\title{
Dynamic Pol expeditions
}

RNA polymerase I is a multicomponent machine that is exclusively responsible for transcribing ribosomal genes in the nucleolus. To conduct its numerous functions, the molecular composition of PolI changes at each step of the transcription cycle (assembly, initiation and elongation). But how do these factors come together at the right time? Reporting in Science last month, Tom Misteli and colleagues (Dundr, M. et al. Science 298, 1623-1626 (2002)) used an elegant combination of in vivo imaging and kinetic modelling to gain new insights into the dynamics of PolI assembly.

To follow the movements of several PolI components in live cells, the authors labelled each factor with green fluorescent protein (GFP). Then, using fluorescence recovery after photobleaching (FRAP), they compared the dynamics of each component (see figure). They found that, whereas pre-initiation factors are rapidly and continuously exchanged with sites of rRNA transcription, a fraction of elongating factors was stably associated with ribosomal genes. Furthermore, each factor showed unique recovery dynamics, suggesting that the components of PolI are imported into the nucleolus as distinct subunits.

Next, the authors used inverse FRAP (in which the entire nucleus, except the site of transcription, is bleached) to see what proportion of each factor is stably associated with engaged PolI and for how long. From the kinetics of these movements, they found that the majority of PolI is not actively elongating at any one time.

Finally, Misteli and colleagues applied a kinetic model of the PolI transcription cycle to the iFRAP data. By altering the parameters of the model so that it fit with the experimental data, they were able to extract quantitative information about the assembly and elongation kinetics of PolI. And what did they learn from all this number crunching? First, they calculated several kinetic properties of PolI, such as in vivo elongation time, elongation rate and the residence time of PolI components at the promoter. They also gained new insight into how PolI assembles. They find that while the recruitment of PolI subunits to the promoter once they enter the nucleolus is high $(50 \%)$, the likelihood of transcription initiation then occurring is remarkably low (1-11\%). Initiation is therefore probably rate-limiting for PolI function. Subunits seem to exchange alone rather than as pre-assembled

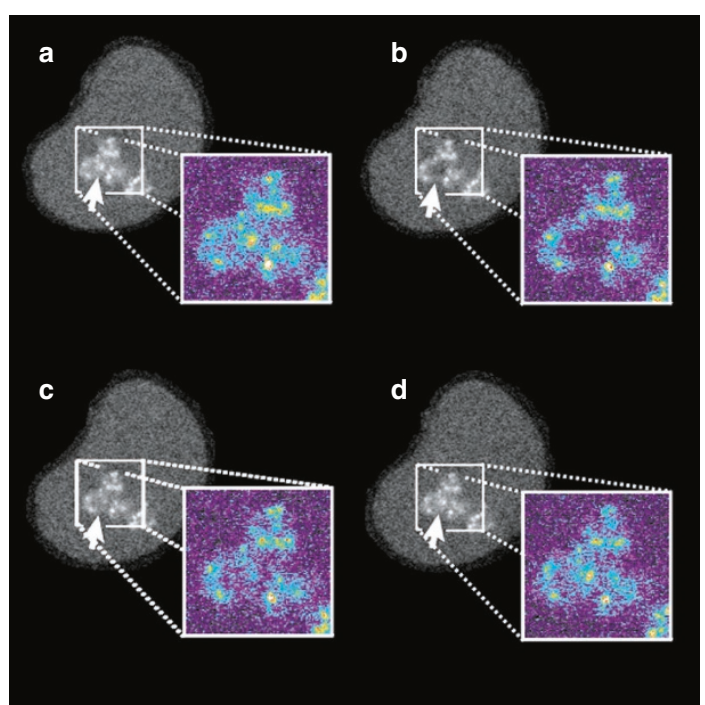

Figure 1 FRAP analysis of the RNA polymerase I transcription factor, upstream binding factor (UBF) fused with green fluorescent protein. The bleached area is indicated by arrows. a shows the cell before bleaching, whereas $b, c$ and $d$ show the cell 0,10 and $60 \mathrm{~s}$ after bleaching, respectively.

complexes and, interestingly, assembly is sequential — possibly involving metastable intermediates - with polymerase stability increasing as more subunits are added.

The wealth of information afforded by these studies demonstrates the combined power of in vivo imaging and computational modelling for dissecting biological processes. The question now is whether the principles that have emerged can be applied to other molecular machines.

ALISON SCHULDT could give rise to an ataxia-telangiectasialike chromosomal instability syndrome. DiTullio et al. offer a more direct link to cancer aetiology with their finding that $53 \mathrm{BP} 1$ is persistently localized into nuclear foci in tumour cell lines lacking p53 (ref. 12). Although the formation of 53BP1 foci and the phosphorylation of Chk2 may not be causally related, these two events were highly correlated in the p53-deficient cell lines. In studies of primary lung and breast tumours, the same investigators report that Chk2 was constitutively phosphorylated at Thr 68 in more than half of the specimens. Thus, activation of ATM and its partners, Chk2 and
53BP1, may be frequent events in human cancer. More work is clearly needed to determine whether constitutive activation of the ATM-dependent checkpoint signalling cascade is causally related to tumour progression, or simply the vestige of a failed effort to cope with the genetic chaos present in many late-stage cancers.

Robert T. Abraham is in The Burnham Institute,

10901 North Torrey Pines Road, La Jolla, CA 92037, USA

e-mail:abraham@burnham.org

\footnotetext{
Iwabuchi, K., Bartel, P. L., Li, B., Marraccino, R. \& Fields, S. Proc. Natl Acad. Sci. USA 91, 6098-6102 (1994).

2. Iwabuchi, K. et al. J. Biol. Chem. 273, 26061-26068 (1998).
}

3. Chai, Y. L. et al. Oncogene 18, 263-268 (1999).
4. Bork P. et al. FASEB J. 11, 68-76 (1997).

5. Derbyshire, D. J. et al. EMBO J. 21, 3863-3872 (2002)

6. Joo, W. S. et al. Genes Dev. 16, 583-593 (2002).

7. Anderson, L., Henderson, C. \& Adachi, Y. Mol. Cell Biol. 21, 1719-1729 (2001)

8. Rappold, I., Iwabuchi, K., Date, T. \& Chen, J. J. Cell Biol. 153, 613-620 (2001).

9. Schultz, L. B., Chehab, N. H., Malikzay, A. \& Halazonetis, T. D. Cell Biol. 151, 1381-1390 (2000).

10. Wang, B., Matsuoka, S., Carpenter, P. B. \& Elledge, S. J. Science 298, 1435-1438 (2002).

11. Fernandez-Capetillo, O. et al. Nature Cell Biol. 4, 993-997 (2002).

12. DiTullio, J., R. et al. Nature Cell Biol. 4, 998-1002 (2002).

13. Zhou, B. B. S. \& Elledge, S. J. Nature 408, 433-439 (2000).

14. Xu, B., Kim, S. T., Lim, D. S. \& Kastan, M. B. Mol. Cell Biol. 22, 1049-1059 (2002).

15. McGowan, C. H. Bioessays 24, 502-511 (2002).

16. Ward, I. M., Wu, X. \& Chen, J. J. Biol. Chem. 276, 47755-47758 (2001). 